\title{
Study of Traffic Sign Detection and Recognition Algorithms
}

\author{
Arasanipalai Mohan Aditya ${ }^{1}$, Sagar Moharir ${ }^{2}$ \\ ${ }^{1,2}$ National University of Singapore, Institute of Systems Science, 25 Heng Mui Keng Terrace, 65162093, Singapore
}

\begin{abstract}
In today's era of autonomous vehicles and connected cars, traffic sign detection and recognition is an important feature of autonomous vehicles and advanced driver assistance systems. There has been extensive research in this field and it has been a consistent application domain in the automotive sector. Recent times have seen the emergence of a large number of public datasets corresponding to different countries, over which traffic sign detection and recognition techniques are being employed. We present a survey paper which involves a comprehensive study of existing techniques being used, along with a performance analysis over different datasets. We also discuss the trade-off of the different options in terms of accuracy and recognition time.
\end{abstract}

Keywords: traffic sign recognition, classification, recognition, road sign

\section{Introduction}

Traffic Sign Recognition systems, in the recent years, has been emerging to the forefront at a substantially high rate. This is driven by the want of intelligent automated systems in the market for various applications such as advanced driver assistance systems, autonomous driving and also the recent boom of public datasets such as Singapore, Thailand and Myanmar.

Having such a system in a vehicle would prove to be tremendously useful. Owing to the increasing traffic and number of accidents which take place due to lack of the driver's concentration on the road, such a system would ensure that the driver concentrates on driving while providing important alerts \& warnings based on the recognized traffic signs. This is because the ability of a driver is largely based on his physical and mental capability which can be affected at any time by drowsiness, emotional stress or any medical condition. Electronic systems on the other hand do not suffer from such issues and hence would prove to be more effective.

Traffic Sign Recognition (TSR) is broadly classified into two problems: Traffic Sign Detection (TSD) and Traffic Sign Classification (TSC) . TSD deals with efficiently localizing the region of interest, the traffic signs, from a real scene. TSC on the other hand deals with classifying the detected traffic sign into its specific category.

The rest of the paper is organized as follows: Section 2 provides details about the different datasets used. Section 3 would largely talk about a detailed survey of different papers and explain the methods of TSD and TSC used within those in detail. Section 4 summarized the performance of various classification algorithms used across different datasets. Section 5 would deal with the major conclusions drawn from this survey paper.

\section{Traffic Sign Datasets}

In this survey paper we talk about TSD and TSC with respect to various datasets: the German Traffic Sign Detection
(GTSD) and Recognition (GTSR) Benchmark, Iranian Road Speed Signs Dataset, Myanmar Traffic Signs Database, the Singapore Traffic Sign Dataset and other small datasets corresponding to the UK, Spain and Czech Republic.

An array of datasets were considered due to the following reasons:

- GTSD and GTSR are very large datasets and have been already subject to a lot of research. Talking about other datasets would expose the public to challenges that still exist.

- Different datasets have some considerable differences in their signs either in terms of classes, color or shape.

- A spread of algorithms can be studied pertaining to various datasets which would provide a more comprehensive idea of existing algorithms.

\section{Detailed Survey}

In this section we will talk about ten research papers in depth, including the image pre-processing stages, feature extraction and classification covering both the TSD and TSC.

In [1], the authors present an optimized method for Iranian road signs detection and recognition. The database consisted of 375 color traffic images with 5 classes for each limit of speeds. The features extracted from these images are used to train the classifier. Image pre-processing and component extraction includes conversion to grayscale, followed by edge detection and then extracting the connected component. Based on the result, the road sign is extracted and noise is removed till the road sign can be clearly seen.

Further, the transit and the angle features are extracted and fed to a Multiclass SVM to be classified. The OAO constructs is used to support the multiclass nature. The system managed to obtain a detection accuracy of $98.66 \%$ and a recognition accuracy of $100 \%$. This has been tested only on still images and is currently being extended to support real time video streams.

Mrinal Haloi [2] talks about a novel method for traffic sign 


\section{International Journal of Science and Research (IJSR) \\ ISSN (Online): 2319-7064}

Index Copernicus Value (2013): 6.14 | Impact Factor (2015): 6.391

recognition using a classification method called probabilistic latent semantic analysis. It is tested using the German sign traffic database. Shape classification and SIFT are initially used to represent the image and detect the traffic sign which is to be classified. pLSA uses a histogram of words concept, wherein each image is converted to a document based on some previously designed vocabulary. Further, the Fuzzy KNN classifier is used to retrieve an appropriate topic for the test image corresponding to the set of training images. 12000 images corresponding to 43 different signs are used. The pLSA method gave an overall recognition accuracy of $98.14 \%$.

This paper [3], presents two biologically inspired approaches to traffic sign recognition, one based on Virtual Generalizing Random Access Memory Weightless Neural Networks (VGRAM WNN) and the other based on the Wilkes, Stonham and Aleksander Recognition Device (WiSARD). Such WNN's are RAM based. RAM-based neural networks, also known as n-tuple classifiers or weightless neural networks, do not store knowledge in their connections but in Random Access Memories (RAM) inside the network's nodes, or neurons. Both approaches have been tested on the German traffic sign database. 51,839 images divided into training and testing are used. A small subset of the images have also been used for tuning the parameters of the WNN. Image preprocessing consists of contrast limited adaptive histogram equalization, filtering using Gaussian filter and localizing the region of interest. Each image is split into its three components ( $\mathrm{R}, \mathrm{G}$ and $\mathrm{B}$ ) and a separate Neural Network is built for each channel. WiSARD WNN was ranked ninth in the GTSRB with a recognition rate of $94.74 \%$, while the VGRAM WNN achieved a higher performance, being ranked fifth in the GTSRB with a recognition rate of $98.42 \%$.

Karla Brki'c in [4], talks about different traffic sign detection methods based on color, shape and learning. Color based extraction basically involves transforming the RGB image to the HIS or L*a*b color space before extraction. Similarly, shape based detectors such as regular polygon detector and the Harris corner detector are used to detect traffic signs. The learning detection method involves used of the Viola \& Jones method. Classification was done based on the Haar-Like classifier and the adaboost ensemble. An overall recognition accuracy of $96 \%$ is obtained.

In [5], Yok-Yen Nguwi et al. target at developing an efficient and intelligent road sign recognition system based on the Singapore traffic sign database. A total of 2500 road and non-road signs have been used for training and testing. The non-road signs have been used to enhance the rejection capability of the system. Binary images are used as part of the system to keep the detection time minimal. Gaussian and laplacian derivatives are used to detect the edge and adaboost is used to remove the unnecessary parts of the image. Critical points in the image are located using the macro and micro SVM based independent component detectors. Gabor wavelets coupled with the Gini feature is used to shrink down the feature set. Support vector machines are used for classification. Using 200 samples for testing, an accuracy of 95\% has been reported.
Yujun Zeng et al. in [6], talk about convolutional neural networks (CNN) as an extractor and extreme learning machines (ELM) as a classifier. The basic dynamically changing parameters which affect the nature of the images are: view point change, color distortion, motion blur, contrast degradation, occlusions and under/over exposure. Initially the image is subjugated to adaptive histogram equalization. The first eight layers of the $\mathrm{CNN}$ is used for feature extraction. The ELM receives input from the feature extractor CNN and is used for classification. The proposed architecture takes about 5-6 hours to train. Current accuracy stands at $99.40 \%$ with claims of increasing it further by equipping the $\mathrm{CNN}$ with blur invariant features.

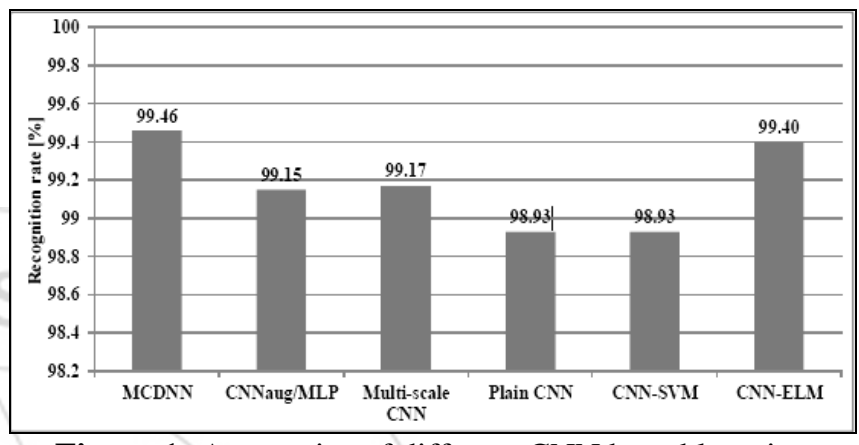

Figure 1: Accuracies of different $\mathrm{CNN}$ based learning algorithms on the GTSRB

In [7], the author presents classification of three signs based on the Malaysian dataset, motor crossing, no entry and stop sign, under different noisy conditions. The traffic sign is detected using shape and color information from the image and by employing background subtraction. A binary pixel based classification method is used wherein the number of pixels of a test image are calculated and matched with the template database. Different illumination and noisy conditions were employed for testing. A summary of the results is in the table below.

\begin{tabular}{|c|c|c|c|c|c|c|}
\hline \multirow[b]{2}{*}{$\begin{array}{l}\text { TRAFFIC } \\
\text { SIGN }\end{array}$} & \multirow[b]{2}{*}{ ANGLE $\left({ }^{\circ}\right)$} & \multirow[b]{2}{*}{ DAYTIME } & \multirow[b]{2}{*}{ BLOCKED } & \multirow[b]{2}{*}{$\begin{array}{c}\text { AFTER } \\
\text { RAINING }\end{array}$} & \multicolumn{2}{|c|}{ NIGHT } \\
\hline & & & & & $\begin{array}{l}\text { ACTUAL } \\
\text { PICTURE }\end{array}$ & $\begin{array}{c}\text { AFTER BEAM } \\
\text { LIGHT (A.LIGHT) }\end{array}$ \\
\hline \multirow{3}{*}{$\begin{array}{l}\text { Motor } \\
\text { Crossing }\end{array}$} & Left $\left(45^{\circ}\right)$ & $95.1 \%$ & $35 \%$ & $80 \%$ & $0 \%$ & $79 \%$ \\
\hline & Centre & $98.9 \%$ & $80 \%$ & $95 \%$ & $0 \%$ & $87 \%$ \\
\hline & Right $\left(45^{\circ}\right)$ & $94.5 \%$ & $30 \%$ & $85 \%$ & $0 \%$ & $90 \%$ \\
\hline \multirow{3}{*}{ No Entry } & Left $\left(45^{\circ}\right)$ & $88 \%$ & $22 \%$ & $22 \%$ & $0 \%$ & $51 \%$ \\
\hline & Centre & $99.97 \%$ & $76 \%$ & $95 \%$ & $0 \%$ & $95 \%$ \\
\hline & Right $\left(45^{\circ}\right)$ & $95 \%$ & $75 \%$ & $81 \%$ & $0 \%$ & $82 \%$ \\
\hline \multirow{3}{*}{ Stop } & Left $\left(45^{\circ}\right)$ & $95 \%$ & $40 \%$ & $70 \%$ & $0 \%$ & $80 \%$ \\
\hline & Centre & $98.7 \%$ & $35 \%$ & $90 \%$ & $0 \%$ & $94 \%$ \\
\hline & Right $\left(45^{\circ}\right)$ & $75 \%$ & $30 \%$ & $56 \%$ & $0 \%$ & $88 \%$ \\
\hline
\end{tabular}

Figure 2: Malaysian Database Results Summary

In [8], the author presents a traffic sign recognition system for roadside images in poor conditions. It deals with the Thailand traffic sign database which is divided into three main classes: prohibitory signs (red and blue signs), general warning signs (yellow signs), and warning signs at construction areas (amber signs). The traffic sign is detected using the following steps: Color (Red, Blue, Yellow and Amber) filtering and segmentation is applied on the image. 


\section{International Journal of Science and Research (IJSR) \\ ISSN (Online): 2319-7064 \\ Index Copernicus Value (2013): 6.14 | Impact Factor (2015): 6.391}

The RGB image is converted into a binary image and the contour of the traffic sign is obtained. Further, the size of the image is checked to verify where the image is traffic sign or not. Traffic sign recognition was done using the particle swarm optimization technique. The test library was formulated using 33 red signs, 19 blue signs, 53 yellow signs and 24 amber signs. The average recognition time was 1.806 seconds. The test results have been summarized in the table below.

Table 1: Thailand Database results summary

\begin{tabular}{|c|c|c|c|}
\hline \multirow{2}{*}{$\begin{array}{c}\text { Type of } \\
\text { Sign }\end{array}$} & \multicolumn{3}{|c|}{ Test Results } \\
\cline { 2 - 4 } & Number of inputs & $\begin{array}{c}\text { Number } \\
\text { Correct }\end{array}$ & $\begin{array}{c}\text { Percentage } \\
\text { Correct }\end{array}$ \\
\hline Red & 36 & 35 & 97 \\
\hline Blue & 27 & 26 & 96 \\
\hline Yellow & 50 & 47 & 94 \\
\hline Amber & 23 & 22 & 96 \\
\hline
\end{tabular}

Wint Sandar Win and Theingi Myint in [9] talk about the traffic sign recognition on the Myanmar dataset. There are four types of traffic signs in Myanmar: prohibitory signs, warning signs, ordered signs and directional (guided) signs. Among these signs, only the first three signs are recognized in this paper. In this paper, although training data are traffic road sign images, testing data are videos of road signs. Some trained images are obtained from google and some are real images. The traffic detection is done by initially converting the RGB model to an HSL model and then applying colour filtering and Harris corner detector to extract the features. In order to recognize the traffic sign the image is converted to grayscale, sobel edge detector is used to find the edges and a template matching algorithm is employed to find the appropriate traffic sign. A detection accuracy of $95 \%$ and a recognition accuracy of $94 \%$ has been obtained by use of this system.

In this paper [10], an algorithm to recognize circular traffic signs has been presented. It follows the methodology as discussed: The traffic sign once detected is converted into a negative image and three vertical and horizontal lines are drawn across the image. The peaks across all these lines are calculated. A peak is defined as a black to white pixel transition. Since we have the number of peaks for all the images, any test image starts off by being divided by one line and then calculating the number of peaks, then narrowing down the probable sign and then proceeding in the same manner till the final sign is determined. To test this system 51 circular traffic signs were used, an average detection time of $8.47 \mathrm{~ms}$ was obtained and the accuracy stands at $100 \%$ owing to the limited dataset.

\section{Performance Analysis}

Now that we have looked at a detailed summary of ten papers, let us look at a graph which summarizes the accuracies of some of the important classification and recognition algorithms used.

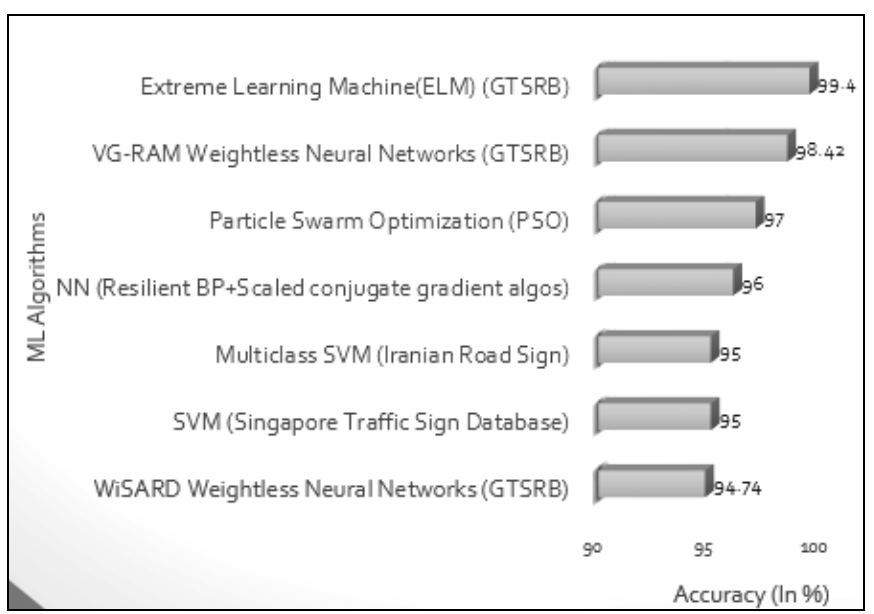

Figure 3: Accuracy summary of important classification and recognition techniques

\section{Conclusions}

After a survey of various papers summarized above there are certain important conclusions which can be drawn. They are:

- Convolutional Neural Networks coupled with Extreme Learning Machines, which forms a complex network structure has an accuracy of $99.40 \%$.

- Having said that, there is still scope for improvement owing to the various inherent noise creating parameters for which optimization techniques are still being researched on.

- There are still unexplored datasets which could lead to new challenges and requirements.

All this is what makes this feature a very active research topic. There is a lot of scope for optimization, which is necessity, since this system is to be deployed in real time environments.

\section{References}

[1] Reza Azad, Babak Azad, Iman Tavakoli Kazerooni, "Optimized Method for Iranian Road Signs Detection and Recognition System". International Journal of Research in Computer Science, 4 (1): pp. 19-26, January 2014. doi: 10.7815/ijorcs.41.2014.077

[2] Mrinal Haloi, A novel pLSA based Traffic Signs Classification System, 2015.

[3] Mariella Berger, Avelino Forechi, Alberto F. De Souza, Jorcy de Oliveira Neto, Lucas Veronese, Victor Neves, Edilson de Aguiar and Claudine Badue, "Traffic Sign Recognition with WiSARD and VG-RAM Weightless Neural Networks," Journal of Network and Innovative Computing, ISSN 2160-2174 Volume 1, pp. 87 - 98, 2013.

[4] Karla Brki'c, "An overview of traffic sign detection methods," Department of Electronics, Microelectronics, Computer and Intelligent Systems Faculty of Electrical Engineering and Computing, Unska 3, 10000 Zagreb, Croatia.

[5] Yok-Yen Nguwi, Teik-Toe Teoh, Insu Song, Siu-Yeung Cho, "Real Time Road Sign Recognition," Nanyang Technological University, Nanyang Avenue, Singapore 639798. 
[6] Yujun Zeng, Xin Xu, Yuqiang Fang, Kun Zhao, "Traffic Sign Recognition Using Extreme Learning Classifier with Deep Convolutional Features," National University of Defense Technology, Changsha, China.

[7] Mohamed Yusof Radzak, "Study on Traffic Sign Recognition," International Journal of Research Studies in Computer Science and Engineering (IJRSCSE), Volume 2, Issue 6, June 2015, PP 33-39, ISSN 23494840 (Print) \& ISSN 2349-4859 (Online).

[8] Thongchai Surinwarangkoon, Supot Nitsuwat, Elvin J. Moore, "Traffic Sign Recognition by Color Filtering and Particle Swarm Optimization," 2012 4th International Conference on Computer Research and Development IPCSIT vol.39 (2012) C (2012) IACSIT Press, Singapore.

[9] Wint Sandar Win, Theingi Myint, "Detection and Recognition of Myanmar Traffic Signs from Video," ISBN 978-93-84468-20-0, Proceedings of 2015 International Conference on Future Computational Technologies, (ICFCT'2015), Singapore, March 29-30, 2015, pp. 150-156.

[10] Khaled M. Almustafa, "Circular Traffic Signs Recognition Using The Number of Peaks Algorithm," Prince Sultan University, Riyadh.

\section{Author Profile}

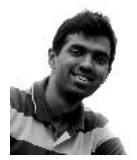

Arasanipalai Mohan Aditya received the B.E. degree in Electronics and Telecommunication Engineering from Pune University in 2012. He is currently pursuing his Masters in Knowledge Engineering from National University of Singapore and is formulating his research in Machine Learning Algorithms for Traffic Sign Recognition in Singapore. His research interests include image processing in advanced driver assistance systems, pattern recognition and machine learning.

\section{Sagar Moharir received the B.E. degree in} Electronics and Telecommunication Engineering from Pune University in 2012 . He is currently pursuing his Masters in Knowledge Engineering from National University of Singapore and is formulating his research in Machine Learning Algorithms for Traffic Sign Recognition in Singapore. His research interests include image processing, computer vision, robotics and machine learning. 